\title{
Die Beschaffung im Rahmen des Portfolio-Managements
}

Das Ziel der Beschaffung in Stadtwerken ist der Einkauf der absatzseitig benötigten Mengen an Gas und Strom. Was an sich nach einer eher simplen Aufgabe klingt, wird jedoch schnell komplex: Welche Mengen werden im folgenden Jahr benötigt? Und in den darauffolgenden Jahren? Und wie sollen diese beschafft werden?

Bei der ersten Frage nach dem „Wie viel“ sind Punkte wie Prognosegüte und Abstimmung mit dem Vertrieb essenziell. Zwischen den Abteilungen Vertrieb und Beschaffung muss eine enge Kopplung hergestellt werden. In vielen Werken geschieht dies in der Form, dass die Verantwortung für beide Bereiche in einer Stelle gebündelt wird: Es existiert ein Leiter Vertrieb/Beschaffung. So kann ein entsprechender Informationsfluss in beide Richtungen sichergestellt werden. Die Informationen von der Beschaffung an den Vertrieb sind eher Gegenstand des Reportings. Informationen über Vertriebsaktivitäten und -erfolge, bzw. allgemein die Entwicklung der Kundenbasis, sind durchaus relevante Punkte für die Beschaffung und müssen regelmäßig aktualisiert und besprochen werden.

Sobald die Frage nach dem „Wie viel“ zufriedenstellend beantwortet ist, muss die Frage nach dem „Wie“ beantwortet werden. Hierauf liegt das größte Augenmerk des Portfolio-Managements. Damit ist man sehr schnell bei der Beschaffung der Mengen am Terminmarkt, da die Zahl der Werke, die sich über Vollversorgungsverträge eindecken, immer weiter abnimmt. Es gilt hier insgesamt, einige wichtige weitere Details festzulegen, auf die im Folgenden noch weiter eingegangen wird. Der Erfolg der Beschaffung spiegelt sich jedoch nicht allein im guten Preis der Terminprodukte im Moment vor Lieferung wider. Auch die Ergebnisse der Spotabwicklung und die Portfoliopreisänderungen durch Ausgleichsenergie müssen hier berücksichtigt werden. Es liegt durchaus in den Händen der Portfolio-Manager, diese Preiskomponenten zu beeinflussen. 